\title{
Erratum to: Phycoerythrin Association with Photosystem II in the Cryptophyte Alga Rhodomonas salina
}

\author{
I. N. Stadnichuk ${ }^{1, a *}$, T. M. Novikova ${ }^{2}$, G. S. Miniuk ${ }^{2}$, V. A. Boichenko ${ }^{3 \#}$, \\ Yu. V. Bolychevtseva ${ }^{4}$, E. S. Gusev ${ }^{1}$, and E. P. Lukashev ${ }^{5}$ \\ ${ }^{1}$ Timiryazev Institute of Plant Physiology, Russian Academy of Sciences, 127726 Moscow, Russia \\ ${ }^{2}$ Kovalevski Institute of Biology of the Southern Seas, Russian Academy of Sciences, 299011 Sevastopol, Russia \\ ${ }^{3}$ Institute of Fundamental Problems of Biology of the Russian Academy of Sciences, 142290 Pushchino, Moscow Region, Russia \\ ${ }^{4}$ Bach Institute of Biochemistry, Research Center of Biotechnology, Russian Academy of Sciences, 119071 Moscow, Russia \\ ${ }^{5}$ Lomonosov Moscow State University, Faculty of Biology, 119991 Moscow, Russia \\ ae-mail: stadnichuk@mail.ru
}

Received April 9, 2020

Revised May 2, 2020

Accepted May 3, 2020

DOI: $10.1134 / \mathrm{S} 000629792008012 \mathrm{X}$

The original article can be found online at https://doi.org/10.1134/S000629792006005X

On p. 679 in the list of authors and affiliations instead of:

${ }^{1}$ Timiryazev Institute of Plant Physiology, Russian Academy of Sciences, 127726 Moscow, Russia

${ }^{2}$ Bach Institute of Biochemistry, Research Center of Biotechnology, Russian Academy of Sciences, 119071 Moscow, Russia

${ }^{3}$ Institute of Fundamental Problems of Biology of the Russian Academy of Sciences, 142290 Pushchino, Moscow Region, Russia

${ }^{4}$ Kovalevski Institute of Biology of the Southern Seas, Russian Academy of Sciences, 299011 Sevastopol, Russia

${ }^{5}$ Lomonosov Moscow State University, Faculty of Biology, 119991 Moscow, Russia

should read:

${ }^{1}$ Timiryazev Institute of Plant Physiology, Russian Academy of Sciences, 127726 Moscow, Russia ${ }^{2}$ Kovalevski Institute of Biology of the Southern Seas, Russian Academy of Sciences, 299011 Sevastopol, Russia

${ }^{3}$ Institute of Fundamental Problems of Biology of the Russian Academy of Sciences, 142290 Pushchino, Moscow Region, Russia

${ }^{4}$ Bach Institute of Biochemistry, Research Center of Biotechnology, Russian Academy of Sciences, 119071 Moscow, Russia

${ }^{5}$ Lomonosov Moscow State University, Faculty of Biology, 119991 Moscow, Russia 